\title{
A comparative study of progabide, valproate, and placebo as add-on therapy in patients with refractory epilepsy
}

\author{
P CRAWFORD, D CHADWICK
}

From the Department of Neurology, Walton Hospital, Liverpool, UK

SUMMARY A three way single blind cross-over comparison of progabide, valproate and placebo, as adjunctive therapy, was undertaken in 64 patients with therapy-resistant partial and generalised seizures. The study was not completed because of the incidence of elevated hepatic enzymes on progabide. Analysis of efficacy showed progabide to be inferior to valproate against all seizure types, particularly against tonic-clonic seizures. Valproate was superior to placebo against all seizure types, partial and tonic-clonic seizures. Progabide did not differ significantly from placebo in any instance. In addition progabide caused elevation of hepatic enzymes which was symptomatic in one case, and was associated with an interaction with phenytoin which resulted in symptoms of intoxication in some cases.

Progabide is a pro-drug and a GABA agonist which possesses anticonvulsant properties in a variety of experimental models of seizures and epilepsy. ${ }^{1}$ Whether it possesses antiepileptic properties in man is controversial. A number of double blind studies against placebo have been reported, some of which are positive $e^{2-6}$ others of which are negative. ${ }^{78}$

One possible reason for negative studies with antiepileptic compounds relates to the selection of patients for the early evaluation of antiepileptic drugs. Patients with frequent seizures, and longstanding epilepsy which has proved resistant to one or more different conventional drugs given either singularly or in combination are usually included. Failure to identify antiepileptic properties of a drug in such patients may not reflect an absence of such properties, but merely that the particular patients studied are incapable of improvement with any presently available compound. This study was undertaken to compare progabide with both placebo and with valproate as a drug of known efficacy in human epilepsy.

\section{Methods}

The trial design was that of a three way cross-over. Each

Address for reprint requests: Dr D Chadwick, Walton Hospital, Rice Lane, Liverpool L9 IAE, UK.

Received 29 November 1985 and in revised form 13 February 1986. Accepted 15 February 1986 treatment limb was of six months with a two week washout and cross-over period between treatment phases. Patients with severe, partial or generalised epilepsies were eligible for admission to the study as long as they had a definite history of epilepsy confirmed by observation and EEG recording and suffered a minimum of one seizure per month during the six months prior to entry into the study. They were taking no more than two conventional antiepileptic drugs and were aged between 15 and 65 years. Women of childbearing potential were advised of the need for adequate contraception during the course of the study. No patients had a progressive neurological disorder and all were thought to be compliant patients who were able to provide adequate seizure diaries. All gave informed consent to the study.

Sixty-four patients were recruited to the study. Randomisation used three by three latin squares. Any drop outs from the study were replaced in order to maintain the balance. It was estimated that a final population of 30-36 patients completing the study would have sufficient power to differentiate between the efficacy of placebo, progabide and valproate.

On entry to the study patients were randomised to receive progabide ( $300 \mathrm{mg}$ tablets) at a daily dose of $30-40 \mathrm{mg} / \mathrm{kg} /$ day or sodium valproate $(200 \mathrm{mg}$ tablets) at a dose of $25-30 \mathrm{mg} / \mathrm{kg} /$ day or placebo in addition to their preexisting antiepileptic therapy. This was achieved over two weeks. Thereafter dosage was increased at each visit by increments of 1 tablet/day in patients showing no clinical response who reported no adverse effects. Median maximum dosage of progabide was $2.4 \mathrm{~g}$ /day and of valproate $1.8 \mathrm{~g} /$ day. Doses of previously administered drugs were maintained throughout the study unless there were clear clinical reasons to alter dosage (eg adverse effects, dimin- 
Table 1 Total exposure to each add-on therapy

\begin{tabular}{|c|c|c|c|}
\hline & Progabide & Valproate & Placebo \\
\hline $\begin{array}{l}\text { Patients* } \\
\text { Days }\end{array}$ & 59 & 56 & 58 \\
\hline Range & $\begin{array}{l}145 \cdot 2 \pm 8 \cdot 2 \\
(15-25 \overline{1})\end{array}$ & $\begin{array}{l}164 \cdot 0 \pm 7 \cdot 0 \\
(7-217)\end{array}$ & $\begin{array}{l}153.8 \pm 7.4 \\
(7-212)\end{array}$ \\
\hline
\end{tabular}

*The total numbers exposed were 62: 59: 60. The numbers presented above, and in tables relating to efficacy are reduced because in 2 or 3 patients adequate data on seizure frequency was not available for a meaningful period of time for all 3 treatments.

ished efficacy associated with falling drug levels of conventional agents).

Serum antiepileptic drug concentrations were recorded at each visit (at least every 2 months) blood being withdrawn approximately 2 hours after morning dosing of drugs. Phenytoin, carbamazepine, primidone and phenobarbitone levels were estimated by the method of Perchalski et al, ${ }^{9}$ valproate levels by the method of Chard ${ }^{10}$ (assays in Liverpool), and progabide and its metabolite levels by the method of Padovani ${ }^{11}$ (assays by LERS, Paris).

It was hoped to conduct the study in a double blind fashion. A number of factors confounded this aim. These included complaints of bright yellow urine in patients receiving progabide, and frequent complaints of gastrointestinal disturbance in patients receiving valproate. (Valproate was provided without enteric coating. A number of patients were subsequently transferred to enteric coated valproate because of this problem.) Following the completion of the first limb of the study a preliminary analysis of results on a parallel group basis was undertaken at the insistence of the company manufacturing progabide and results presented at a symposium. Thus in practice this became a single blind study.

The trial design was not completed as planned because of the withdrawal of clinical trial exemption by the regulatory authority during the final phase of the study, because of the occurrence of elevated liver enzymes in patients receiving progabide (see below). In spite of this sufficient patients were exposed to the different treatments to warrant analysis of efficacy. The total duration of exposure of patients to each treatment is shown in table 1.

\section{Patient population}

Sixty-four patients were recruited to the study, 38 male and 26 female. Ages ranged between 17 and 57 years (mean $31 \cdot 5$ ), the duration of epilepsy ranged from 7-51 years (mean $18 \cdot 5$ ). Forty-six were taking two antiepileptic drugs on admission

Table 2 Drop-outs and early cross-over

\begin{tabular}{llll}
\hline & Progabide & Valproate & Placebo \\
\hline Administrative & 11 & 6 & 4 \\
Lack of efficacy & 3 & 0 & 12 \\
Adverse events & 5 & 6 & 0 \\
Patients request & 3 & 0 & 1 \\
\hline
\end{tabular}

All patients including drop-outs have been included in analysis of adverse effects. to the study and 28 were taking a single agent. Forty-one patients received phenytoin ( $150-500 \mathrm{mg}$ per day), 48 carbamazepine (300-1600 mg per day), 13 phenobarbitone (30-240 $\mathrm{mg}$ per day), three received primidone $(500-1225 \mathrm{mg}$ per day) four clonazepam, and one diazepam. Forty-five patients had partial epilepsies. Seven patients had simple partial seizures with secondary generalisation, 11 had only complex partial seizures, and 27 had complex partial seizures with secondarily generalised attacks.

Nineteen patients had generalised epilepsies. This was idiopathic in seven patients who all had tonic-clonic seizures. Five patients had symptomatic tonic-clonic seizures alone. In six patients such attacks were combined with complex absence or atonic seizures, and in one patient myoclonic seizures occurred in addition to tonic-clonic seizures.

Thirty of the 64 patients had an identified aetiology responsible for their epilepsy. In nine this related to perinatal brain damage, in six to febrile convulsions, in five the epilepsy was post encephalitic and in eight it was post traumatic. Eighteen patients had neurological signs or intellectual deficit.

\section{Results}

\section{(a) Efficacy}

Details of patients dropping out from the study or crossing over prematurely from one treatment limb to the next are given in table 2 . The most common reason for failure to complete a limb was an administrative one, that is, due to the premature termination of the trial following reporting of elevated transaminase to the regulatory authorities. However five patients withdrew from the study without participating in one or two limbs. Two patients never took progabide, five patients never took valproatec and four patients never took placebo. In two cases (included in the previous figures) both valproate and placebo were not taken.

No patients receiving valproate crossed over at an early stage because of lack of efficacy but three patients receiving progabide and 12 patients receiving placebo crossed over prematurely for this reason.

Because of the premature termination of the study and the varying periods for which patients received the test treatments all subsequent comparisons relate to mean four weekly seizure frequencies derived for each patient who received all three therapies.

Non-parametric analysis of variance showed that differences between varying sequences of administration of drugs were not significant $(p=0.56)$. However the power of this observation is low and there is some evidence that two particular sequences showed a high seizure rate throughout, that is placeboprogabide-valproate and valproate-progabideplacebo. As it is unlikely that any such differences arose other than by chance it seems reasonable to proceed to an anlysis of seizure frequencies in three treatment periods for all patients receiving all three 
Table 3 Seizures/4 weeks by treatment seizure type

\begin{tabular}{|c|c|c|c|c|}
\hline & & Progabide & Valproate & Placebo \\
\hline $\begin{array}{l}\text { Tonic-clonic }(n=46) \\
\text { Partial }(n=33) \\
\text { All seizures combined }(n=51)\end{array}$ & $\begin{array}{l}\text { Median } \\
\text { Range } \\
\text { Median } \\
\text { Range } \\
\text { Median } \\
\text { Range }\end{array}$ & $\begin{array}{l}1 \cdot 18 \\
(0-20) \\
3 \cdot 15 \\
(0-109) \\
8 \cdot 78 \\
(0-402 \cdot 6)\end{array}$ & $\begin{array}{l}0.74 \\
(0-17) \\
3 \cdot 20 \\
(0-67 \cdot 8) \\
4 \cdot 85 \\
(0 \cdot 14-326)\end{array}$ & $\begin{array}{l}1 \cdot 5 \\
(0-21) \\
6 \cdot 68 \\
(0-80 \cdot 2) \\
11 \cdot 58 \\
(0 \cdot 29-292 \cdot 8)\end{array}$ \\
\hline
\end{tabular}

treatments. All statistical comparisons are "within patient".

Table 3 presents data on seizure frequency in 51 patients receiving all three treatments for a minimum 4 week period.

The figure illustrates seizure frequency on each active therapy compared with placebo within individual patients for tonic-clonic seizures, partial seizures and all seizures. Overall $24 \%$ and $37 \%$ of patients were improved by more than $50 \%$ by progabide and valproate respectively, $10 \%$ and $14 \%$ by greater than $75 \%$. Two patients had no seizures on progabide, but both received the drug for only 1 month, and their prior seizure frequency was low (approximately $1 /$ month). The same trend towards greater improvement overall on valproate is seen when par- tial seizures or tonic-clonic seizures are considered separately. It is evident that the main difference between the two drugs appeared to be that larger numbers of patients receiving progabide were worse or unchanged (placebo frequency $\pm 20 \%$ ) than on valproate.

Considering the changes in frequency of all seizure types within patients there was a significant difference between the treatments (Friedman non parametric analysis of variance, $p=0.0003)$. Valproate was significantly superior to placebo $(p=0.0001)$ and to progabide $(p=0.005)$. There was no significant difference between progabide and placebo $(p=0.42)$. These latter comparisons used Wilcoxon non parametric tests. When only tonic-clonic seizures were considered there was again a significant difference
All seizures

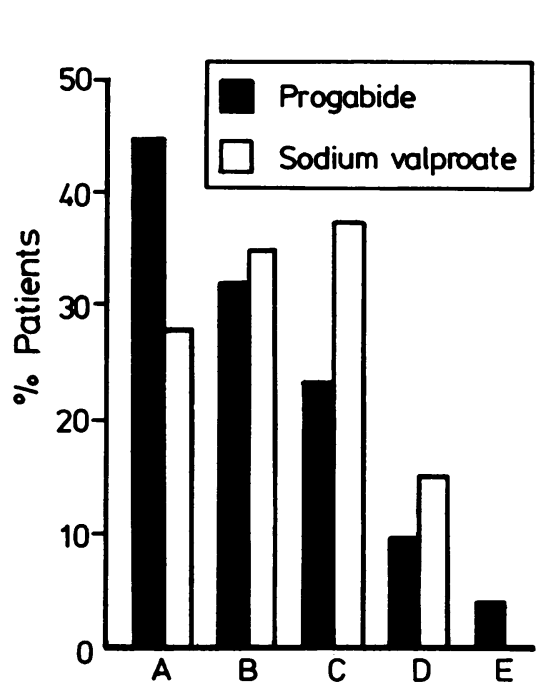

Partial seizures

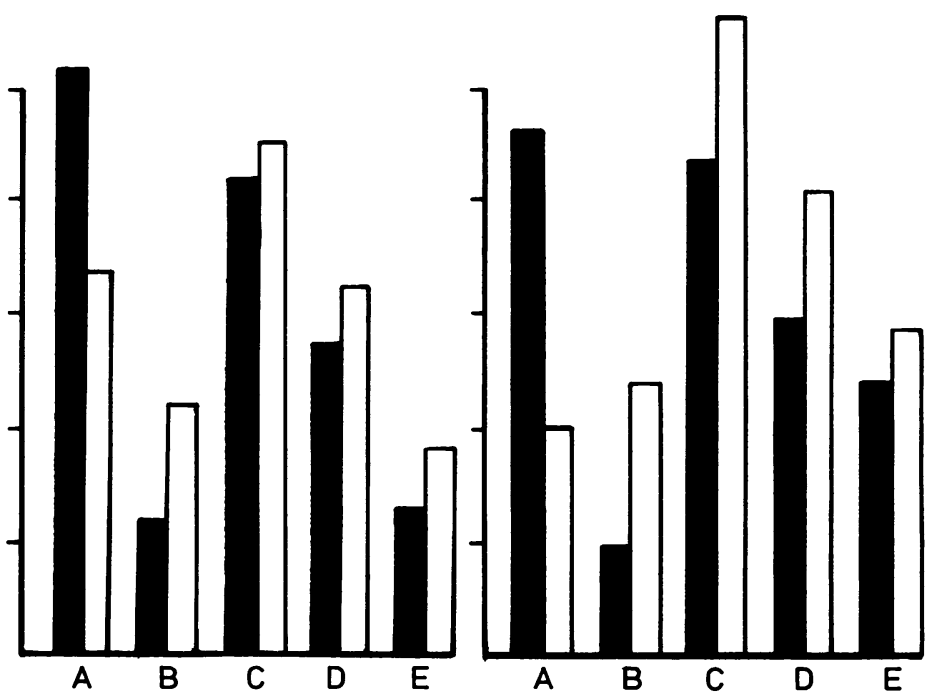

Tonic clonic seizures
Fig Distribution of percentage change in 4 weekly seizure frequency on valproate and progabide when compared to placebo periods for total seizures, partial seizures only, and tonic-clonic seizures only. (Within patient comparisons.) $A=$ worse or unchanged ( $<20 \%$ improved), $B=20-49 \%$ improvement, $C=50-74 \%$ improvement, $D=75-99 \%$ improvement, $E=$ seizure free. 
between treatments $(p=0.0035$, Friedman test). Valproate was significantly superior to placebo $(\mathrm{p}=$ $0.0001)$ and to progabide $(p=0.009)$. The difference between progabide and placebo was not significant $(p$ $=0.40$ ). There was a less significant difference between treatments for partial seizures $(p=0 \cdot 03)$. Valproate was significantly superior to placebo ( $\mathrm{p}=$ $0.005)$. However there was no difference between valproate and progabide $(\mathrm{p}=\mathbf{0 . 7 3})$ or between progabide and placebo $(\mathrm{p}=0.38)$.

Some sub-group analyses were undertaken. There was no evidence that patients with partial or generalised epilepsies differed in their responses, and results were uninfluenced by whether or not the patient was taking phenytoin, offering no support to the hypothesis that any antiepileptic effect of progabide is purely due to interaction with phenytoin.

\section{(b) Adverse effects}

The incidence of reported adverse events is summarised in table 4 and the type of these symptoms in table 5. Adverse events were more commonly reported on active drug than on placebo. CNS symptoms are reported in more detail in table 6 . There was an undoubted excess of dizziness, ataxia, and diplopia in patients receiving progabide (in combination with phenytoin). These symptoms were associated with elevated phenytoin levels which necessitated reduction in phenytoin dosage in nine patients during the progabide limb of the study. Tremor occurred in six patients receiving valproate. Nausea and vomiting was the commonest gastrointestinal complaint. Although this was reported in a similar number of patients on progabide and valproate (7 and 9) it was only so troublesome as to lead to premature crossover

Table 4 Incidence of adverse events

\begin{tabular}{|c|c|c|c|}
\hline Patients & Progabide & Valproate & Placebo \\
\hline \multirow{3}{*}{$\begin{array}{l}\text { No treated } \\
\text { No with } 1 \text { or more } \\
\text { adverse events } \\
\text { Total adverse } \\
\text { reports }\end{array}$} & 62 & 59 & 60 \\
\hline & $55(88 \cdot 7 \%)$ & $41(69 \cdot 5 \%)$ & $34(56 \cdot 7 \%)$ \\
\hline & 157 & 100 & 56 \\
\hline
\end{tabular}

Table 5 Summary of adverse events

\begin{tabular}{lccc}
\hline & Progabide & Valproate & Placebo \\
\hline Nervous system & 113 & 67 & 40 \\
Gastrointestinal & 16 & 17 & 6 \\
Urinary tract & 8 & 2 & 0 \\
"Pains" & 5 & 2 & 0 \\
Skin and appendages & 4 & 7 & 5 \\
Miscellaneous & 11 & 5 & 5 \\
Total & 157 & 100 & 56 \\
\hline
\end{tabular}

Table 6 Nervous system events

\begin{tabular}{lrrr}
\hline & Progabide & Valproate & Placebo \\
\hline Behavioural problems/ & & & \\
bad tempered/instable & 6 & 5 & 2 \\
Drowsy, tired, slow, & 35 & 22 & 13 \\
$\quad$ apathetic & 6 & 4 & 3 \\
Confused & 14 & 1 & 2 \\
Dizziness & 12 & 3 & 2 \\
Unsteady/ataxic & 11 & 6 & \\
Diplopia & 2 & 2 & \\
Blurred vision & 3 & 3 & 4 \\
Anxiety/tension & 4 & 1 & 1 \\
Nightmares, dreams, & voices, etc & 6 & \\
Tremor & & & \\
\hline
\end{tabular}

in patients receiving valproate (which was not enteric coated in this study).

There were two significant skin rashes. One patient receiving progabide developed a Stevens-Johnson syndrome, and one patient receiving valproate developed erythema multiforme. Both patients recovered uneventfully on withdrawal of the drug. One patient receiving valproate reported hair loss. Complaints of dysuria and frequency occurred in eight patients taking progabide but only two receiving valproate.

In six patients adverse effects necessitated either hospital admission or time off work. One patient receiving valproate was admitted to a gastrointestinal unit for investigation of nausea and vomiting. On cessation of valproate therapy, she improved and was discharged. Four patients receiving progabide had muscle and joint pains, and two dropped out from the study for this reason. All settled on withdrawal of progabide and did not occur on rechallenging. The remaining patient developed clinical hepatitis on progabide therapy, and was off work for ten weeks.

\section{Laboratory investigations}

Screening of haemoglobin, red cell and white cell and platelet counts, $\gamma$-GT, SGOT, SGPT, bilirubin, alkaline phosphatase, urea and electrolytes, creatinine, serum protein and blood glucose were undertaken at at least 2 monthly intervals. There were no significant haematological changes during the study.

Changes in SGOT and SGPT are summarised in

Table 7 SGOT and/or SGPT during the study

\begin{tabular}{llll}
\hline & Progabide & Valproate & Placebo \\
\hline $\begin{array}{l}\text { No patients with } \\
1 \text { or more tests }\end{array}$ & 56 & 55 & 55 \\
$\begin{array}{l}\text { No patients with 1 test } \\
1-2 \text { times ULN }\end{array}$ & 7 & 0 & 1 \\
No patients with 1 test & 7 & 0 & 0 \\
$>2$ times ULN & 7 & &
\end{tabular}


table 7. Seven patients receiving progabide showed significant elevation of these liver enzymes (more than twice upper limit of normal). In one patient this was symptomatic. She received $37.5 \mathrm{mg} / \mathrm{kg} /$ day of progabide during the third phase of the study. SGOT and SGPT were normal at 24 days. Thirty-one days later she was seen with symptoms suggestive of a phenytoin intoxication. Seventeen days later, and following reduction of her dose of phenytoin she complained of anorexia, vomiting and lethargy. SGOT was $1364 \mathrm{IU} / 1$ and SGPT was > $300 \mathrm{IU} / 1$. Progabide was withdrawn and 12 days later SGOT and SGPT were $900 \mathrm{IU} / 1$. The patient's symptoms improved and liver function tests returned to normal. In two patients elevation of SGOT levels was detected at the end of a 6 month period of progabide treatment. In both cases liver function tests returned to normal. Two patients showed elevation of SGOT levels at the three month visit which subsequently settled during continued progabide therapy. One patient showed elevation of SGOT at one, three and six months (104 to 144 IU/1). SGOT settled to normal values on the next limb of the therapy. One patient who discontinued progabide because of a Stevens-Johnson syndrome had an SGOT of $124 \mathrm{IU} / 1$ at the time. Values subsequently reverted to normal. $\gamma$-GT levels were greater than twice the upper limit of normal in $39 \%$ of patients on progabide, $23 \%$ of patients on sodium valproate and $20 \%$ of patients taking placebo. No significant changes in other biochemical variables were noted during the study.

\section{(c) Compliance and drug therapy}

There was evidence of satisfactory compliance throughout this study. Progabide serum levels ranged between 115 and $4219 \mu \mathrm{g} / \mathrm{ml}$. In seven patients progabide blood levels were found to be less than $1000 \mu \mathrm{g} / \mathrm{ml}$ on at least one occasion. Levels of the metabolite (SL76102) were similarly satisfactory. Serum valproate concentrations ranged between 5 and $114 \mu \mathrm{g} / \mathrm{ml}$. Fifteen patients had levels of valproate less than $50 \mu \mathrm{g} / \mathrm{ml}$ on at least one occasion.

Overall there were no significant changes in serum concentrations of phenytoin, carbamazepine and other anticonvulsant drugs in the three treatment periods. In 35 instances phenytoin concentrations of less than $10 \mu \mathrm{g} / \mathrm{ml}$ were recorded, and in 18 cases concentrations were greater than $20 \mu \mathrm{g} / \mathrm{ml}$.

There was, however, undoubted evidence of interaction between phenytoin and progabide in this study. In nine patients dosage of phenytoin was reduced during progabide therapy, compared with one patient during valproate therapy and one patient during placebo. Tinis action was necessary because of symptoms of phenytoin intoxication which were usually associated with blood levels greater than $20 \mu \mathrm{g} / \mathrm{ml}$.

\section{Discussion}

Progabide can claim to be the first potential antiepileptic agent to be developed on sound scientific principles, its GABA agonist activity being compatible with the GABA deficiency theory of epilepsy. It was unfortunate that this comparative study of progabide could not be completed and the results analysed as originally planned. The failure of all patients to be exposed to three treatment limbs for similar periods of time has led to some difficulties in the analysis of efficacy, but the results are of interest because of the large number of patients studied, and the long period of treatment.

The results of this study are disappointing. There was a trend towards improvement on progabide and $25 \%$ of patients were improved by $50 \%$ or greater on progabide compared to placebo. The present study must, however, be added to other studies which have failed to find a statistically significant difference between the efficacy of progabide and placebo. ${ }^{78}$

Whilst it may be argued that a failure to show a statistically significant difference between the efficacy of progabide and placebo against tonic-clonic seizures, partial seizures or all seizure types could be related to the severe nature of the epilepsies in our population, we have shown a significant improvement with valproate (used as an antiepileptic agent of known efficacy). In our patients valproate is significantly superior to placebo in the treatment of partial seizures, tonic-clonic seizures and all seizure types. It does therefore seem that the group of patients we have studied can be benefited by an additional anticonvulsant agent. Indeed it is of interest that valproate appears effective as adjunctive therapy in patients with partial epilepsies. This is in keeping with its effects as sole therapy in previously untreated patients with partial epilepsy, which are comparable to those of phenytoin. ${ }^{12}$

It cannot be argued that our negative findings for progabide are a result of a low power of our observations, as approximately twice as many patients as were required, were recruited to the study. What is remarkable is the ability of this study to differentiate clearly between the efficacy of valproate and progabide. Comparative studies of antiepileptic agents are few, but only one, a study comparing phenytoin and sulthiame has ever previously demonstrated a difference in efficacy between two putative antiepileptic drugs. ${ }^{13}$ One other comparison of valproate and progabide has been undertaken. ${ }^{14}$ Preliminary results included small numbers of patients and had insufficient power to differentiate between the drugs, 
although a trend towards greater improvement with valproate was evident.

Progabide, as well as appearing less efficacious than valproate also presented greater problems in usage. The most worrying aspect is the observed hepatotoxicity in our patients. This has been reported in other studies. ${ }^{15}$ SGOT levels were raised to more than twice the upper limit of normal in seven of 62 patients exposed to progabide. Whilst only one patient had symptoms directly related to these disturbances, these observations led to the termination of the study when reported to the British regulatory authorities. Whilst a full clinical trial certificate has now been granted for further investigations into progabide in the United Kingdom the relatively high percentage of patients developing raised transaminases $(7.1 \%$ of 561 patients in Europe, and $5.8 \%$ of 121 cases in the United States, ${ }^{16}$ and clinical hepatitis (five cases, including one death from hepatic failure, ${ }^{16}$ excluding our own case)) would seem likely to limit the use of this drug when no preferential antiepileptic properties compared to valproate can be demonstrated.

A further matter complicating the use of assessment of progabide is its interaction with phenytoin. In this study nine patients required reduction of phenytoin dosage during treatment with progabide because of the development of symptoms of phenytoin intoxication. This emphasises the clinical importance of this interaction which has been reported in other studies. ${ }^{1517}$ The apparent ability of progabide to lower carbamazepine concentrations was not observed in this study. ${ }^{717}$

In conclusion therefore we have failed to demonstrate a statistically significant antiepileptic effect of progabide in this study which provides strong evidence that progabide is less effective than valproate and had greater toxicity and interaction with other antiepileptic drugs.

We are grateful to LERS, Synthelabo for financial support of this study and statistical analysis. We thank our colleagues for allowing us to study their patients.

\section{References}

${ }^{1}$ Lloyd KG, Zivkovic B, Scatton B, Bartholini G. Evidence for functional roles of GABA pathways in the mammalian brain. In: Bowery NG, ed. Actions and Interactions of $G A B A$ and Benzodiazepines. New York: Raven Press, 1984:59-79.

${ }^{2}$ Martinez-Lage JM, Bossi L, Morales G, Martinez Vila E, Orofiamma B, Viteri C. Progabide treatment in severe epilepsy: a double blind cross-over trial versus placebo. Epilepsia 1984;25:586-93.
${ }^{3}$ Loiseau P, Bossi L, Guyot M, Orofiamma B, Morselli PL. Double-blind cross-over trial of progabide versus placebo in severe epilepsy. Epilepsia 1983;24:703-15.

${ }^{4}$ Kulakowski S, Meynckens M, Coupez-Lopinot $\mathbf{R}$. Double-blind study of a new antiepileptic drug, progabide in severe childhood epilepsy. In: Bartholini G, Bossi L, Lloyd KG, Morselli PL, eds. Epilepsy and GABA Receptor Agonists: Basic and Therapeutic Research. New York: Raven Press, 1985:377-88.

${ }^{5}$ Van der Linden G, Meinardi H, Meijer J, Bossi L, Gomeni R. A double-blind cross-over trial with progabide (SL76002) against placebo in patients with secondary generalised epilepsy. In: Dam M, Gram L, Penry J, eds. Advances in Epileptology. 12th Epilepsy International Symposium. New York: Raven Press, 1981:141-4.

${ }^{6}$ Weber M, Vespignani H, Remy MC, Regnier F, Bossi L. Controlled trial of progabide versus placebo in severe therapy-resistant epilepsies. In: Bartholini G, Bossi L, Lloyd KG, Morselli PL, eds. Epilepsy and GABA Receptor Agonists: Basic and Therapeutic Research. New York: Raven Press, 1985:353-62.

${ }^{7}$ Dam M, Gram L, Philbert A, Hansen BS, et al. Double blind study of progabide in severe epileptics suffering from complex partial seizures. In: Bartholini G, Friedmann JC, Langer SZ, Morselli PL, eds. Epilepsy and GABA Receptor Agonists: Basic and Therapeutic Research. New York: Raven Press, 1985:327-34.

${ }^{8}$ Schmidt D, Utech K. Progabide as adjunctive therapy in patients with complex partial seizures refractory to high-dose antiepileptic drugs. In: Bartholini G, Friedmann JC, Langer SZ, Morselli PL, eds. Epilepsy and GABA Receptor Agonists: Basic and Therapeutic Research. New York: Raven Press, 1985:363-7.

${ }^{9}$ Perchalski RJ, Scott KN, Wilder RJ, Hammer RH. Rapid simultaneous GLC determination of phenobarbital, primidone and diphenyhydantoin. $J$ Pharm Sci 1973;62:1735-6.

${ }^{10}$ Chard CR. A simple method for the determination of Epilim in serum. In: Legg $\mathbf{N}$, ed. Clinical \& Pharmacological aspects of sodium valproate (Epilim) in the Treatment of Epilepsy. Tunbridge Wells: MCS Consultants, 1967:89-91.

${ }^{11}$ Padovani P, Deves C, Bianchetti G, Thenet JP, Morselli PL. Determination of progabide and its main acid metabolite in biological fluids using HPLC and electrochemical detection. Application to the measurement of blood to plasma partition ratio. $J$ Chromatogr 1984;308:229-39.

12 Turnbull DM, Howel D, Rawlins MD, Chadwick DW. Which drug for the adult epileptic patient: phenytoin or valproate. Br Med J 1985;290:815-9.

${ }^{13}$ Chadwick D, Turnbull DM. The comparative efficacy of anticonvulsant drugs for partial and tonic-clonic seizures. J Neurol Neurosurg Psychiatry 1985;48:1073-7.

${ }^{14}$ Bossi L, Beaussart M, Courjon J, et al. Multicentre study of progabide versus sodium valproate in therapy resistant epilepsy. In: Bartholini G, Friedmann JC, Langer SZ, Morselli PL, eds. Epilepsy and GABA Receptor Agonists: Basic and Therapeutic Research. New York: Raven Press, 1985:415-20.

15 Van Parys JAP, Van der Linden GJ, Goedhart DM, Meijer JWA, Meinardi H. Clinical Experience with Pro- 
gabide in severe in and out patients. In: Bartholini G, Friedmann JC, Langer SZ, Morselli PL, eds. Epilepsy and GABA Receptor Agonists: Basic and Therapeutic Research. New York: Raven Press, 1985:343-52.

${ }^{16}$ Coquelin JP, Krall R, Bossi L, Musch B, Morselli PL. Drug safety profile of progabide. In: Bartholini $G$, Friedmann JC, Langer SZ, Morselli PL, eds. Epilepsy and GABA Receptor Agonists: Basic and Therapeutic
Research. New York: Raven Press, 1985:431-40.

${ }^{17}$ Cloyd JC, Brundage RC, Leppik IE, Graves NM, Welty TE. Effect of progabide on serum phenytoin and carbamazepine concentrations: a preliminary report. In: Bartholini G, Friedmann JC, Langer SZ, Morselli PL, eds. Epilepsy and GABA Receptor Agonists: Basic and Therapeutic Research. New York: Raven Press, 1985:271-7. 